\title{
Deadlock Avoidance in Manufacturing Systems with a Multiple Resource Request Model
}

\author{
Robert P. Judd \\ School of Electrical Engineering \\ and Computer Science \\ Ohio University \\ Athens, $\mathrm{OH} 45701$
}

\author{
Wenle Zhang \\ School of Electrical Engineering \\ and Computer Science \\ Ohio University \\ Athens, $\mathrm{OH} 45701$
}

\author{
Robert Lipset \\ Department of Industrial and \\ Manufacturing Systems Engineering. \\ Ohio University \\ Athens, $\mathrm{OH} 45701$
}

\begin{abstract}
A deadlock avoidance method for flexible manufacturing systems with both free choices and multiple resource requests allowed in part routing is presented. Based on a digraph model of the system, the method classifies whether moving a part to its next step is safe, unsafe, or undetermined. An undetermined part movement is further analyzed using a very efficient system simulation, which attempts to empty the system virtually to determine whether the move is safe. This classification algorithm is shown to be polynomial in complexity. To avoid deadlocks, only safe part movernents should be allowed to proceed. An example is provided to illustrate how the method can be applied.
\end{abstract}

\section{INTRODUCTION}

In the past decade, considerable research has been performed on the topic of developing deadlock control policies for flexible manufacturing systems. Some of the significant works have adopted Petri net models as a formalism to describe a manufacturing system. Banaszak and Krogh [1] proposed a deadlock avoidance algorithm (DAA) that developed a restriction policy to guarantee that no circular wait situations would occur. Viswanadham et al. [9] developed a deadlock avoidance algorithm that used a look-ahead policy. Structural properties of PNs such as siphons are used in $[2,4]$ to determine potential deadlock situations.

Another formalism is to describe the manufacturing system using graphs. In this approach, the vertices represent resources and the edges represent part flows between resources. Cho et al. [3] developed the concept of bounded circuits with empty and non-empty shared resources to detect deadlock. Fanti et al. [5] developed a simple graph-theoretic method for deadlock detection and recovery in systems with multiple capacity resources. Judd and Faiz [6] derived a set of static linear inequalities that, when satisfied, avoid deadlock. Lipset et al. [8] expanded upon [6], and quantified both necessary and sufficient conditions for deadlock to occur in a manufacturing system. Lipset et al. [7] developed a polynomial complexity deadlock avoidance algorithm by classifying part movements.

Very few papers are found to have studied the deadlock avoidance problems with a multiple resource request model in the manufacturing context. Reveliotis et al. [10] developed a deadlock avoidance policy for a conjunctive resource allocation model by extending their previous result based on the resource upstream neighborhood concept, where a conjunctive resource allocation model is a multiple resource request model and a resource vector is used to represent multiple resource requests in a process plan.

In this paper, we will extend [7] to allow flexible manufacturing systems with both free choices and multiple resource requests in process plans (FCMRR). Section 2 discusses the FCMRR model and properties. Section 3 reviews and modifies the classifications. Section 4 presents the new algorithm, and Section 5 gives an example using the proposed algorithm.

\section{FCMRR SYSTEM MODEL}

A FCMRR system consists of a set $R$ of resources such as machines, robots, AGVs, etc., and a set $P$ of product types. Each resource has a capacity, denoted as $C \mathrm{r} \geq 1$, which can be considered as $C r$ identical units. At any time, the system contains a set $Q$ of parts. Each part $q$ belongs to a product type $p \in P$ and each product type $p$ possesses a process plan that describes the resource sequence required to produce it. We assume that a process plan is of finite length. A process plan consists of process steps. In a FCMRR system, a process step can be: i) a simple step that requests exactly one unit of a specific resource; ii) a free choice step that requests one resource unit out of a subset of resources (the OR [11] resource allocation model), or iii) a multiple resource request (MRR) step that requests one unit from each resource in a subset of resources (the AND [11] model). By free choice in a process plan, we mean that the part's next resource can be arbitrarily chosen from the subset of resources and the system controller will dispatch the part to the chosen resource. While at a MRR step, a part needs the exclusive use of all the resources in the subset. The implication is that we need to redefine an "enabled" part and the deadlock state of the system.

\subsection{The Wait Relation Graph}

A FCMRR system can be described by a digraph, called a wait relation graph (WRG), which consists of a set of nodes to represent resources of the system and a set of arcs to represent individual process sequences for all product types.

For a choice step, we draw an arc (labeled with a '+') from the current resource to every resource in the subset. When the system is simulated, a part can be simply moved to the resource chosen by the system controller.

For a MRR step, we also draw an arc (labeled with a ' $\bullet$ ') from the current resource to every resource in the subset. When the system is simulated, a part will be split to all the resources in the subset. Naturally, we will also have a converse operation; that is, to merge a set of part splits. If every part split's next resource is the same, then these splits can be merged at the next resource.

A path in a wait relation graph $G$ is a set of nodes that are connected to one another within the graph. A closed path starts and ends on the same node. A simple circuit is a closed path with no repeated nodes, except for the common starting and ending node. A semi-closed path is a path that is not closed but contains a closed path. The portion of a semi-closed path that is not on the contained closed path is a tail. The resource that connects the tail and the closed portion is the key resource.

\subsection{Process Plan Representation}

Our method to represent a process plan with choices is to "straighten" the plan. Simple steps are listed resource by resource sequentially, and steps that have choices are listed branch by branch sequentially. Each branch further consists of sequences of resources and other branches in an iterative manner. In this way, each process plan can still be represented as a sequence of resources. To unify the various types of process steps, we

\section{U.S. Government work not protected by U.S. Copyright 4443}


decompose a MRR step. That is, we consider each resource of a MRR step as a separate simple step/branch, as in the choice case, and we split the part into every resource of the MRR step. In this way, our method allows a process step to acquire/release resources while keeping some current resources. The method associates each process step with a step type: $s-$ for simple, $c-$ for choice and $m$-for MRR. In addition to the sequence of resources, our method also associates each process plan with three data structures: i) $T$, a vector that specifies the step type; ii) $N$, a vector that counts the number of branches (choice/MRR) at each step; and iii) $B$, a matrix that identifies the next step(s), where each step has a corresponding column of the matrix. With choices, a process plan can have more than one terminal step. All terminal steps are given the same next step number-0. Several functions are defined. Function len $(q)$ returns the plan length of part $q$. Function branches $(q, n)$ returns the number of branches at step $n$ of the plan of part $q$. Function step_type $(q, n)$ returns the type of the step as one of simple, choice or MRR. Function next $(q, n, b)$ returns the next step of part $q$ after step $n$ on branch $b$. See the example in Section 5.

\subsection{Deadlock In A FCMRR System}

Normally, a flexible manufacturing system would start from an empty state with no parts in the system; that state is usually referred to as the idle state.

Each part $q$ has a current step $n(0 \leq n \leq \operatorname{len}(q))$, where $n=0$ means the part is waiting to be loaded into the system or has exited the system. A system state is a vector of dimension $\Sigma \mathrm{Cr}$, $\forall r \in R$, denoted as $s=[n: q$ 's current step, $\forall q \in Q \mid 0$ for empty resource units]. The idle state is denoted as $s_{0}$.

A part is enabled if its next resource has an empty unit for a simple step, one of its next resources has an empty unit for a choice step or all of its next resources have an empty unit for a MRR step. An enabled part can be moved into its next resource(s). A state $s_{\mathrm{j}}$ is reachable from state $s_{\mathrm{j}}$ if there exists a sequence of part movements and the system state changes from $s_{j}$ to $s_{\mathrm{i}}$ after the sequence of part movements; otherwise, it is unreachable.

A system state $s_{\mathrm{j}}$ is called a deadlock if state $s_{0}$ is unreachable from $s_{i}$. Deadlock states can be further categorized into two major types: i) a primary deadlock state in which there exist parts in a circular wait situation which can involve parts at a choice or MRR step; and ii) an impending deadlock state which is not a primary deadlock state, but must evolve into a primary deadlock state in a finite number of part movements. The algorithm to be presented herein will be able to efficiently avoid both types of deadlocks.

\section{CLASSIFICATIONS OF PART MOVES}

In the following, we will divide part movements into two major categories, i.e. live moves and dead moves. See Figure 3.1. A dead move will cause a system in a non-deadlock state to enter a deadlock state, and a live move will not cause a non-deadlock system to enter a deadlock state. Our algorithm categorizes a large class of moves as live in linear time. These moves are termed safe moves. The algorithm also identifies a class of dead moves, which are termed unsafe, through the introduction of the canonical semi-closed path concept. Moves that cannot be determined to be either safe or unsafe are classified as undetermined. Those will be analyzed further through a virtual system simulation.

When the manufacturing system is in any particular state $s$, there usually exists an enabled part. Let $s^{\prime}$ denote the state of the system after an enabled part is moved. An enabled part must be analyzed to determine whether the move will result in deadlock The following subsections summarize the eight classes of part

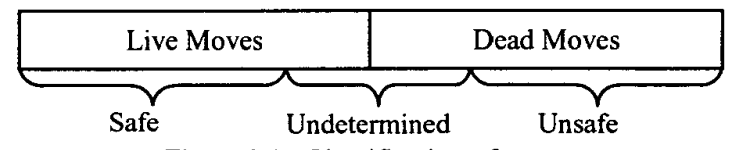

Figure 3.1. Classification of moves

movements developed in [7] along with appropriate modifications to accommodate the multiple resource request model.

\subsection{Basic Classes}

There are three classes of safe moves that can be determined immediately by examining the current and next resources in the process plan.

Class 1. Advancing a part/split that is at its last step is safe.

Class 2. Advancing a part/split onto a non-converging resource (one that has only one input arc) is safe.

Class 3. Advancing a part/split that does not move onto any new closed paths is safe.

Proof. The same as in [7].

\subsection{Dynamic Subgraph (Path)}

To categorize other classes of part moves, it is necessary to build a dynamic subgraph. A dynamic subgraph is formed by concatenating the resources in the plan of the candidate part until a different part is encountered. The subgraph is continued by appending the resources of that new part's process plan. When a part at a choice/MRR step or a multiple capacity resource with a part is seen, the dynamic subgraph will be split into several branch paths. If the resource is free (not filled to capacity), then a special path corresponding to the part entering the resource is also constructed. That part is called the ghost part with respect to this resource because the resource does not actually contain the part. Branch paths are also constructed for all the parts contained in the resource.

For each part, there are three possible types of paths that can exist in such a dynamic subgraph:

i) an open path,

ii) a closed path that includes the original part, and

iii) a semi-closed path in which the original part is on the tail.

Paths on the dynamic subgraph are terminated when i) the terminal resource of the plan for the current part is reached, ii) a part is encountered for the second time (PST), or

iii) a resource is encountered for the second time (RST).

\subsection{Canonical Semi-Closed Path}

A canonical semi-closed path (CSC) is a semi-closed path with a part on the tail that enters the closed portion of the CSC, and a part on the closed portion that leaves the CSC through its key resource. This concept is developed to detect/avoid impending deadlocks. Details can be found in [7].

\subsection{More Classes For Part Moves}

Based on the dynamic subgraph, several other classes can be determined. In the following, let $q$ be the candidate part, $r_{0}$ be the resource that contains $q$ and $r_{1}$ be a next resource.

Class 4. Advancing a part/split that has a free exit is safe. A free exit means that all resources needed by the rest of the part's process plan have at least one free unit.

Proof. Same as in [7].

Class 5. Let $S_{\mathrm{CSC}}$ be the set of all moves for a CSC such that the pivot part has not yet entered the key resource. Then moving the initiating part on the CSC into its key resource is a class 5 move if it deadlocks the closed portion of the CSC for all moves in $S_{\mathrm{CSC}}$. Since, by definition, the pivot part must leave the system through the key resource, a class 5 move is a dead move and will 
be classified as unsafe.

For the purpose of classifying part moves, a CSC can be reduced by replacing the closed portion of the CSC with only the key resource [7]. More complicated class 5 moves can be analyzed through the process of CSC reduction. This reduction will be performed logically by the algorithm introduced later. The system graph will not be physically reduced.

The remaining three classes are all undetermined. They can not be classified as either safe or unsafe from only the information developed by building the dynamic subgraph. See [7] for details.

Class 6 (undetermined_0), is a part that moves onto a closed path that contains the part, but is neither a class 2 nor a class 3 .

Class 7 (undetermined_1), is a part on an open path but is not a class 4 move

Class 8 (undetermined_1), is a part that is on the tail of a semiclosed path, but does not fall into any other class.

\subsection{A New Class Related to MRR}

A new class will be identified based on a MRR step. This class is safe. The class is numbered $2 b$ because the move does not involve any closed path examination.

Class $2 b$. Advancing a part split whose next step is $n_{1}$ to a resource that contains a split $n_{2}$ of the same part will cause the two splits to merge, and is, therefore, safe.

Proof. Once this type of move is made, the part will occupy no further resource space but will release the resource space it is occupying.

\section{DEADLOCK AVOIDANCE ALGORITHM}

The modified method to determine whether advancing a candidate part results in a live state or not is presented in the following three algorithms: dynamo_MRR, empty_system and live. Dynamo_MRR performs the classification, empty_system performs the virtual system simulation and live is the main function. For a part at a choice step, the best class (if we order from best to worst as safe, undetermined, and unsafe) among all branches should be chosen since the system controller can determine which choice to select. On the other hand, for a part at a MRR step, the worst classification should be chosen, which means the part is safe only if the part is safe on every branch, and the part is unsafe if the part is unsafe on any branch.

\subsection{Dynamic Path Status}

Each of the stopping conditions is associated with a status value. Dynamo_MRR examines all possible paths in a dynamic subgraph and keeps track of the one with the best-suited status. It then makes conclusions on whether the proposed move is live or not based on this optimal path within the dynamic subgraph.

1) PST. A PST status is defined when a part is seen for the second time. PST means that a part was found on either a closed dynamic path or on a semi-closed path.

2) RST. An RST status is defined when a free resource is encountered for the second time, indicating a (component) CSC was found. The resource is the key resource. The part leading the path to the RST resource for the first time is the initiating part of the CSC. The part leading the path to the RST resource for the second time is the pivot part. Associated with an RST status is the space flag used to determine whether the space in the key resource is needed to avoid deadlock. A false space flag means that the key resource was seen for the second time without seeing any other free resource after the first time it is seen. This means moving the initiating part into the key resource is a class 5 move for this component CSC. After all component CSCs sharing the same key resource have been found, the space flags of all component CSCs are ORed/ANDed together. If they are all class 5 moves, then algorithm dynamo declares that the entire CSC is a class 5 move.

3) EXIT. This is the best possible status. EXIT status means either the candidate part or another part can exit. Even though EXIT as a status is better than RST, the algorithm gives higher priority to detect RST over EXIT unless the candidate part is at its terminal step. The reason is to make sure that the dynamic path has enough space to accommodate the candidate part, so that the part that is found to exit actually can exit. A part at a choice step is given an EXIT status as long as EXIT is found on one of the branches. On the other hand, a part at a MRR step receives an EXIT status only if EXIT is found on every branch.

To help build the dynamic subgraph, an AND-OR tree will be created and expanded while the dynamic subgraph is being constructed. The AND-OR tree is composed of nodes. A node is recursively defined with a set of child nodes or (sub) trees,

$$
\mathrm{C}=\text { (type, status, part, space, }\left\{\mathrm{C}_{1}, \mathrm{C}_{2}, \ldots, \mathrm{C}_{\mathrm{k}}\right\} \text { ), }
$$

where: i) type is the type of the node; ii) status is the classification status at the node; iii) part is the part corresponding to the status; iv) space is the space flag to indicate whether free space is available or not on the dynamic path; v) $k$ is the number of child nodes and $C_{\mathrm{i}}, i=1 \sim k$, is a child node. The set of child nodes is empty only for a terminal node. An internal node is created only for a part at a choice/MRR step and for a non-empty multiple capacity resource. The tree can be graphically represented.

There are three node types:

i) END for a terminal node, represented as a blank oval;

ii) OR for a choice step or a non-empty multiple capacity resource, represented as an oval with a "+" in it;

iii) AND for a MRR step, represented as an oval with a " $\bullet$ " in it.

There are four values for node status:

i) EXIT for a part that sees a free exit;

ii) PST for a part that is seen for the second time;

iii) RST for a free resource that is seen for the second time;

iv) UNSAFE for the root node that is concluded unsafe.

The node status is set according to the stopping condition for terminal nodes and can be calculated for internal nodes as follows (using the above order),

status $=\left\{\begin{array}{l}\text { the lowest child status, for an OR node } \\ \text { the highest child status, for an AND node }\end{array}\right.$

The space is set to FALSE for an RST terminal node when it is created, TRUE for an EXIT or PST because no CSC is involved and is calculated for an internal node as in the following,

space $=\left\{\begin{array}{l}\text { logical OR of child spaces, for OR nodes } \\ \text { logica }\end{array}\right.$

The space can become TRUE when the algorithm returns to a free resource that has multiple capacity or is not a key resource.

\subsection{The Stop-Check-and-Go}

The stop-check-and-go mechanism is performed when status RST is detected. This mechanism implements the CSC reduction.

The stop. If the path encounters a resource for the second time that has at least one free unit (RST), then: i) the path stops; ii) the last part (the pivot part) that would have entered the key resource and its process step are recorded in tree $C$, and; iii) returns to the point when the key resource was visited for the first time.

The check. As the algorithm returns to the key resource, any resources with free capacity will be flagged.

The go. When the algorithm returns to the key resource, the flag indicates whether space on the CSC can be used. If the flag is true, all the pivot parts in $C$ will be chased further to determine which one will produce the optimal path. Otherwise, the algorithm continues to returm. 


\subsection{Algorithm dynamo_MRR}

Algorithm dynamo_MRR consists of a group of procedures: dynamo_MRR, check_resource and check_part. The algorithm defines for class $2 b$ a separate status, called MERGE, for use in the main procedure dynamo_MRR and for virtual system simulation only. MERGE is a class of safe moves.

The algorithm first checks whether the move is one of the first four classes of safe moves. If none of those classes applies, then the algorithm starts the construction of the dynamic subgraph which is stored in AND-OR tree $C$ by calling check_resource. Check_resource and check_part form an indirect recursion. Check_resource calls check_part for every part in the resource, including the ghost part, and check_part calls check_resource for the next resource on each branch. Because of this recursive nature, the construction of the dynamic subgraph consists of two stages: the forward process and the backward process (processing after returning from a recursive call).

As the construction of the dynamic subgraph progresses forward, the algorithm tracks the free resources it encounters in $R$ to check for a RST condition and the parts it sees in $Q$ to check for a PST condition. During the backward process, in addition to filling internal nodes with child nodes, the algorithm checks whether any free resources can be used by a component CSC.

The go of the stop-check-and-go is also implemented in the backward process. It actually starts a new forward process, called a sub-forward process, which takes the form of a sub-tree and eventually replaces the corresponding RST terminal node. This sub-forward process can be considered the continuation of the main forward process. Since the main forward process never sees the sub-forward process, to get a correct combined AND-OR tree, the parent nodes' data needs to be adjusted based on the sub tree. The adjustment of status is made to every ancestor node until the adjusted status of a node remains unchanged, or the root node is reached. The adjustment of space is also made to every ancestor node until the adjusted space remains unchanged or the root node is reached. Figure 4.1 illustrates the adjustment process.

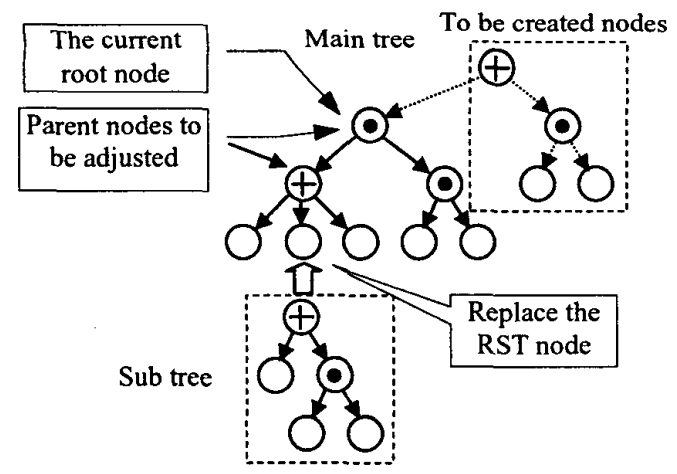

Figure 4.1 Sub tree and status/space back propagation

\subsubsection{Listing of dynamo_MRR}

Let $\mathrm{q} 0$ be the candidate part

if $q 0$ is at a simple step then

if $\mathrm{q} 0$ belongs to one of the first four classes then return the class

else call check_resource on next resource $\mathrm{r} 1$ to construct the AND-OR tree $C$ and return the class found else ( $q 0$ is at a choice/MRR step)

for next resource $r 1$ on each branch do

if $q 0$ belongs to one of the first four classes then record the class

else call check_resource on $\mathrm{r} 1$ to construct the AND-OR tree $\mathrm{C}$ and record the class

end for

return the best/worst class for choice/MRR end

4.3.2 Listing of check_part

if the part being checked is at a simple step then

if the part is at the last step then

create an END node $C$ with status EXIT

else call check_resource to construct a sub tree C

else (the part is at a choice/MRR step)

create an OR or AND node $C$

for next resource $r$ on each branch do

if the part is at the last step then

create a child END node Ch with status EXIT

if a choice step then break out the for loop

else call check_resource on $r$ to construct a sub tree $\mathrm{Ch}$ and add child node $\mathrm{Ch}$ to $\mathrm{C}$

$$
\text { end for }
$$

set C's status to the best/worst of child node status

set C's space to the logical OR/AND of child node space end

\subsubsection{Listing of check_resource}

1. The forward process

let $q$ be the part being checked

let $r$ be the resource being checked

if $r$ is in $R$ then

create an END node $C$ with status RST and return

if $r$ is free then (ghost part)

add $r$ to $R$, call check_part on $q$ to construct a sub tree $\mathrm{Ch}$

if $r$ has multiple capacity and is not empty then

create an OR node $\mathrm{C}$ and

if $\mathrm{Ch}$ is created then add child node $\mathrm{Ch}$ to $\mathrm{C}$

if $r$ is not empty then

for each part $p$ in $r$ do

if $p$ is in $Q$ then

create an END node $C$ with status PST and return

else add $p$ to $Q$, call check_part on $p$ to construct a sub-

tree $C h$ and if $C$ is created then add child node $C h$ to $C$ end for

if $C$ is created then

set C's status to the best of child node status

set C's space to the logical OR of child node space

else set $\mathrm{C}$ to $\mathrm{Ch}$ (single capacity or empty resource)

2. The stop and check

if $r$ is free but has a FALSE space flag then

if $r$ is not an key resource or $r$ has more than 1 free space or ( $r$ has multiple capacity and $|R|>1$ ) then set C's space to TRUE

if C's status is RST and space is FALSE and |R| is 1 then update C's status to UNSAFE and return

3. The go

while $C$ has an RST node and the key resource is $r$ do let $p$ be the pivot part

call check_part on $p$ to construct a sub tree $\mathrm{C} 1$ replace the RST node with $\mathrm{C} 1$ and update all parent nodes' status and space

end while

end 


\subsubsection{Analysis of dynamo_MRR}

The following theorem establishes that the algorithm dynamo_MRR terminates and has linear complexity.

Theorem 4.1. The complexity of dynamo_MRR is linear in the system size, $L=\operatorname{sum}(\operatorname{len}(p)), \forall p \in P$.

Proof. The same as of dynamo in [7].

The following lemma will help to establish the correctness of algorithm live.

Lemma 4.2. If algorithm dynamo_MRR classifies a part move as safe, then the proposed move is a live move.

Proof. We will show this result in the following three cases:

If the candidate part is at a simple step, then the proposed move is classified as safe only if it belongs to either the first four classes (class 1,2,2b and 3 ) or class 4 .

If the candidate part is at a choice step, then the proposed move is classified as safe only if on one of its branches it belongs to either the first four classes (class 1, 2, 2b and 3) or class 4.

If the candidate part is at a MRR step, then the proposed move is classified as safe only if on every branch it belongs to either the first four classes (class $1,2,2 \mathrm{~b}$ and 3 ) or class 4 .

According to choice/MRR definitions and proofs for classes 1, $2,2 b, 3$ and 4 , the proposed move is indeed a live move

\subsection{Algorithm empty_system}

Given a set of parts $Q$ in state $s$, empty_system attempts to find a set of moves that will remove all parts from the virtual system using only safe moves. It invokes algorithm dynamo_MRR on every enabled part. Empty_system will execute all the safe moves first. Parts returned with status EXIT from dynamo will be taken out of the virtual system immediately. Parts returned with status MERGE or SAFE will be merged or advanced and the set of enabled parts will be re-evaluated. If only undetermined_0 moves exist, then it will arbitrarily pick one and proceed. The algorithm continues this cycle until it empties all parts from the system; it then returns true. It returns false if the system cannot be emptied. It will be shown that the algorithm has polynomial complexity.

\subsubsection{Listing of empty_system}

let $Q$ be the set of parts in the system

while $Q$ is not empty do

let $E$ be the set of enabled parts, $E \subseteq Q$

call dynamo_MRR to classify each part $p \in E$

if any part classified as exit, safe, merge or

undetermined_0 then

remove all parts classified as exit from $Q$

advance/split all parts classified as merge

if there is a part classified as safe then

advance/split the part

else if there is a undetermined_ 0 part then

advance/split the part

else return false (system can not be emptied)

end while

return true

end

\subsubsection{Analysis of empty_system}

The following result shows that the algorithm has polynomial complexity.

Theorem 4.3. The complexity of algorithm empty_system is $\mathrm{O}\left(L^{3}\right)$, where $L=\operatorname{sum}(\operatorname{len}(p)), \forall p \in P$.

Proof. The same as in [7].

\subsection{Algorithm live}

Among the three algorithms, live is the algorithm to be called first to determine whether a proposed move is live. It further invokes dynamo $M R R$ to perform the classification. Its complexity is linear in $L$ unless dynamo returns undetermined, in which case it will invoke empty_system and the complexity is therefore $\mathrm{O}\left(L^{3}\right)$. Live begins by classifying the proposed part move. A virtual move is made if the proposed move is found to be undetermined. Live then calls empty_system to try to find a way to empty the virtual system to determine whether the original proposed move is indeed live. The algorithm is listed below.

\section{Listing of live}

Let $q$ be the proposed part

call dynamo_MRR to classify $q$

if $q$ is classified as exit or safe then return true else if $q$ is classified as unsafe then return false else (undetermined) advance $q$ and return empty_system end

Theorem 4.4. If algorithm live returns TRUE (classifies the part move as safe), then the proposed move is a live move.

Proof. Live returns TRUE only in the following two cases:

i) dynamo classifies the part as exit or safe;

ii) dynamo classifies the part as undetermined, the virtual move is made and empty_system returns TRUE to indicate it can find a sequence of moves to empty the system.

In case $\mathrm{i})$, the proposed move is a live move according to Lemma 4.2. In case ii), since empty_system can empty the system, the proposed move must be a live move.

The above theorem establishes the correctness of algorithm live. Unfortunately, the algorithm does not provide optimality. In a few cases, it will be possible for live to return false for a live move. This will happen if, while emptying the virtual system, a state is reached where no enabled parts are safe moves, multiple undetermined_0 moves are detected, and the undetermined_0 move that is chosen results in deadlock of the virtual system, whereas selecting a different undetermined_0 move would not have.

\section{EXAMPLE}

An example illustrating choices in process plans was given in [7]. In this section, we will present an example system with MRR steps in the process plans.

Example 5.1. Let the manufacturing system be composed of three resources $r_{1}, r_{2}$, and $r_{3}$ : All resources are of unit capacity. Suppose the system manufactures two types of products, $p_{\mathrm{A}}$ and $p_{\mathrm{B}}$, specified by the following process plans: $p_{\mathrm{A}}=r_{1} r_{2} r_{3}, p_{\mathrm{B}}=r_{3} r_{2} r_{1}$. After applying phase I of [2], that is to add the 3 control places to control the 3 minimal uncontrolled siphons identified $\left(\left\{\mathrm{p}_{\mathrm{A} 2}, \mathrm{p}_{\mathrm{B} 3}\right.\right.$, $\left.\mathrm{r}_{1}, \mathrm{r}_{2}\right\},\left\{\mathrm{p}_{\mathrm{A} 3}, \mathrm{p}_{\mathrm{B} 2}, \mathrm{r}_{2}, \mathrm{r}_{3}\right\}$ and $\left.\left\{\mathrm{p}_{\mathrm{A} 3}, \mathrm{p}_{\mathrm{B} 3}, \mathrm{r}_{1}, \mathrm{r}_{2}, \mathrm{r}_{3}\right\}\right)$, an augmented Petri net is obtained as in Figure 5.1. However, it is known that this controlled net can still run into deadlock. Now let's apply our method to the augmented system. Part of type $p_{\mathrm{A}}$ will need first $r_{1}, c_{1}$ and $c_{3}$, then $r_{2}, c_{2}$ and $c_{3}$, finally $r_{3}$. The process plan is given in Table 5.1 .

Table 5.1. Process plan for $p_{\mathrm{A}}$

\begin{tabular}{|l|ccccccc|}
\hline step No. & 0 & 1 & 2 & 3 & 4 & 5 & 6 \\
\hline resource list & $\mathrm{r}_{0}$ & $\mathrm{r}_{1}$ & $\mathrm{r}_{2}$ & $\mathrm{r}_{3}$ & $\mathrm{c}_{1}$ & $\mathrm{c}_{2}$ & $\mathrm{c}_{3}$ \\
\hline step type T & $\mathrm{m}$ & $\mathrm{m}$ & $\mathrm{s}$ & $\mathrm{s}$ & $\mathrm{m}$ & $\mathrm{s}$ & $\mathrm{s}$ \\
\hline $\mathrm{N}$ & 3 & 2 & 1 & 1 & 2 & 1 & 1 \\
\hline $\mathrm{B}$ & 1 & 2 & 3 & 0 & 2 & 3 & 3 \\
& 4 & 5 & & & 5 & & \\
\hline
\end{tabular}


Parts of type $p_{\mathrm{B}}$ will need first $r_{3}, c_{2}$ and $c_{3}$, then $r_{2}, c_{1}$ and $c_{3}$, finally $r_{1}$. The process plan can be given similarly.

Suppose part $a$ of type $p_{\mathrm{A}}$ is already loaded, as shown in Figure 5.2, that is we have three part splits $a_{1}, a_{2}$ and $a_{3}$. We want to know whether introducing part $b$ of type $p_{\mathrm{B}}$ is live. We first call live. Live calls dynamo_MRR to classify part $b$ and dynamo_MRR will check for part $b$ along each of its 3 branches beginning with $r_{3}, c_{2}$ and $c_{3}$, respectively. The branch with $r_{3}$ will return a class 5 through building the AND-OR tree, see the tree built in Figure 5.3.

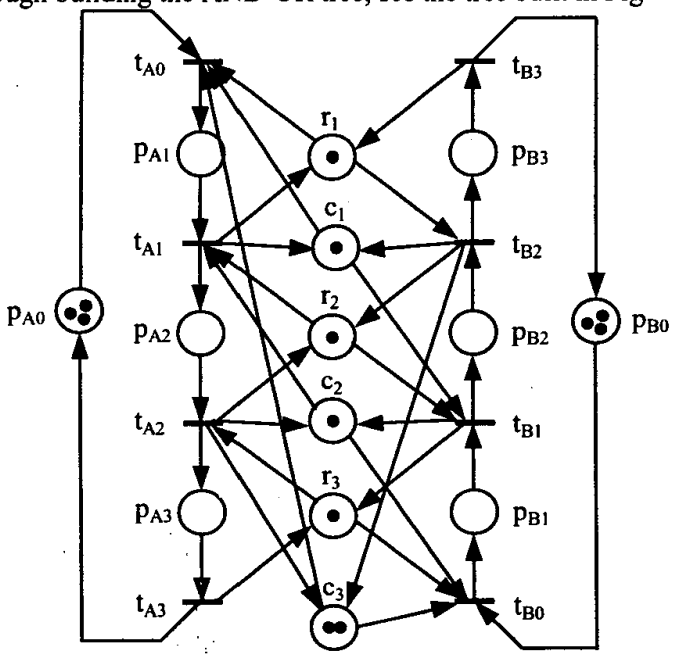

Figure 5.1 Petri net for example 5.1

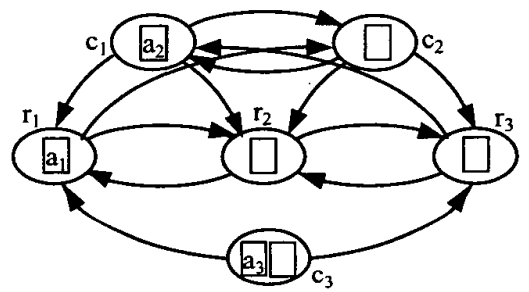

Figure 5.2 The WRG for example 5.1

The branch with $c_{2}$ will return a class 5 similarly and the branch with $c_{3}$ will return a class $7\left(a_{1}\right.$ can exit). So, dynamo_MRR will return the worst class, which is a class 5 . Therefore, loading part $b$ should be avoided.

Note if applying dynamo_MRR to the original system without the added three control places, then the algorithm can run much more efficiently because of no MRR steps, avoid all the deadlock states and keep all live states.

\section{DISCUSSION}

To improve our algorithm, the change would involve trying all undetermined_0 moves if no safe moves existed, instead of selecting one arbitrary move of this type. If that change is made, however, live may no longer terminate in polynomial time. A counter could be used in empty_system to limit the number of searches of undetermined 0 moves. This counter would then become a tuning parameter to trade off the algorithm's complexity versus the degree of completeness to which the algorithm can determine all live moves. One of future research subjects is to extend our method to include the AND/OR resource request model [11].

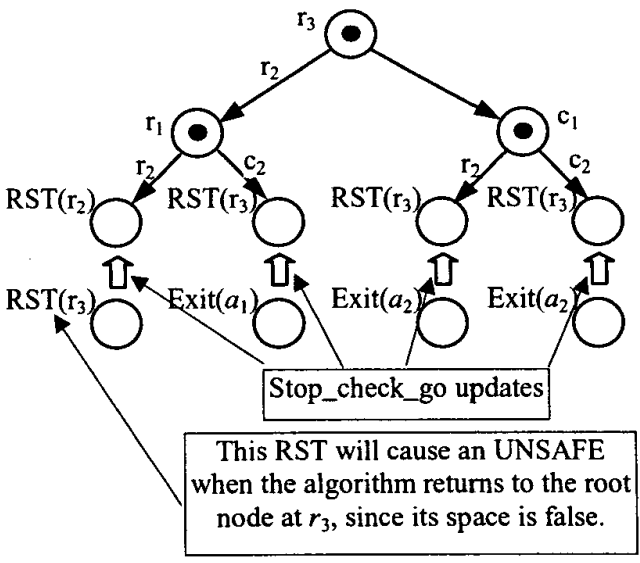

Figure 5.3 The AND-OR tree built for example 5.1

\section{REFERENCES}

[1] Banaszak, Z. and B. Krogh, "Deadlock Avoidance in Flexible Manufacturing Systems with Concurrently Competing Process Flows." IEEE Trans. Robotics \& Auto. 6, no. 6, 724-733.

[2] Barkaoui, K., and 1. B. Abdallah, "Deadlook Avoidance in FMS Based on Structural Theory of Petri Nets," IEEE Symp. Emerging Tech. \& Factory Automátion, 499-510.

[3] Cho, H., T.K. Kumaran, and R. Wysk, ""Graph-Theoretic Deadlock Detection and Resolution for Flexible Manufacturing Systems," IEEE Trans. Robotics \& Auto. 11, no. 3, 550-527.

[4] Ezpeleta, J., J. M. Colom, and J. Martinez, "A Petri Net Based Deadlock Prevention Policy for Flexible Manufacturing Systems," IEEE Trans. Robotics \& Auto. 11, no. 2, 173-184.

[5] Fanti, M., G. Maione and B. Turchiano, "Deadlock detection and recovery in flexible production systems with multiple capacity resources," Proc. of the 1996 Mediterranean Electrotechnical Conference, 237-241.

[6] Judd, R. P. and T. Faiz, "Deadlock Detection and Avoidance for a Class of Manufacturing Systems." Proceedings of the 1995 American Control Conference, 3637-3641.

[7] Lipset, R., R. P. Judd and W. Zhang, " Deadlock Avoidance in Manufacturing Systems with Non-Deterministic Part Routings", Proceedings of the 2001 American Control Conference, 49304936.

[8] Lipset, R., P. Deering, and R. P. Judd, "Necessary and Sufficient Conditions for Deadlock in Manufacturing Systems," Proc. of the 1997 American Control Conference, 1022-1026.

[9] Viswanadham, N., Y. Narahari, and T. Johnson, "Deadlock Prevention and Deadlock Avoidance in FMS Using Petri Net Models," IEEE Trans. on Robotics and Auto. 6, no. 6, 713-723.

[10] Reveliotis S., M. Lawley and P. Ferreira, "Polynormial Complexity Deadlock Avoidance Policies for Sequential Resource Allocation Systems", IEEE Trans. Automatic Control, 42(10): 1344-1357, 1997.

[11] Singhal, M. and N. Shivaratri, "Advanced Concepts in Operating Systems", McGraw-Hill, Inc., 1994 